\title{
Carnets
}

Revue électronique d'études françaises de l'APEF

Deuxième série - 6 | 2016

Exotopies de Barthes

\section{Barthes en espace urbain}

Maria Hermínia A. Laurel

\section{(2) OpenEdition}

\section{Journals}

Édition électronique

URL : http://journals.openedition.org/carnets/740

DOI : $10.4000 /$ carnets. 740

ISSN : 1646-7698

Éditeur

APEF

\section{Référence électronique}

Maria Hermínia A. Laurel, « Barthes en espace urbain », Carnets [En ligne], Deuxième série - 6 | 2016, mis en ligne le 31 janvier 2016, consulté le 19 avril 2019. URL : http://journals.openedition.org/ carnets/740; DOI : 10.4000/carnets.740

Ce document a été généré automatiquement le 19 avril 2019.

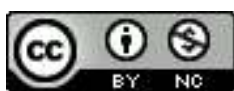

Carnets est mis à disposition selon les termes de la licence Creative Commons - Atribution - Pas d'utilisation commerciale 4.0 International. 


\title{
Barthes en espace urbain
}

\author{
Maria Hermínia A. Laurel
}

\section{NOTE DE L'AUTEUR}

Cet article s'insère dans la recherche menée au sein du Programme Stratégique UID/ ELT/00500/2013 (Instituto de Literatura Comparada Margarida Losa, Faculté des Lettres de l'Université de Porto).

Un beau texte est comme une eau marine ; sa couleur vient du reflet de son fond sur sa surface, et c'est là qu'il faut se promener, et non dans le ciel ou dans les abîmes.

(Roland Barthes, « Réflexion sur le style de L'Etranger, de Camus », 1944.

1 Intellectuel sensible aux tendances de la contemporanéité, sinon les déterminant luimême, Roland Barthes a participé à la réflexion sur le phénomène urbain qui faisait se rejoindre architectes, historiens de la ville et d'autres penseurs, à partir de la décennie de 1960. La conférence qu'il donne en 1967 sous le titre "Sémiologie et urbanisme $»^{1}$ en témoigne. Travailler sur Barthes en espace urbain m'a ainsi semblé intéressant, dans la mesure où cela constituait une invitation à des sentiers éventuellement moins diffusés de la réflexion barthésienne. Devant la richesse des aspects évoqués par Barthes dans l'article qui donna lieu A la conférence citée, et face à l'intertexte que cet article interpelle "Barthes en espace urbain» me suggère à son tour plusieurs voies de recherche, que plusieurs sous-titres auraient pu indiquer. Ce sont bien souvent les soustitres qui orientent les choix de lecture. LA pensée de Barthes est une «pensée ouverte »

Les sous-titres que nous allons proposer essayeront de rendre compte de ces possibilités de lecture, de cette ouverture.

3 Ainsi, un premier sous-titre nous permettrait de considérer Roland Barthes comme lecteur de la ville en sémiologue, en « amateur de signes » et de la ville, selon ses propres mots. Un second, comme lecteur de la ville en poète ${ }^{2}$, déçu, selon A. Compagnon qui le 
présente en antimoderne, se tournant dans son dernier cours au Collège de France « de plus en plus vers la poésie comme seule chance de sauver la littérature dans un monde qui ne l'aime plus (...) qui l'ignore de plus en plus»(Compagnon, 2005 : $404 ; 436$ ); Barthes, pour qui « seul le poème [pouvait] encore racheter la littérature, lui rendre vie et sauver le monde » (ibid. : 436). Un poète qui, dans Leçon, célébrait la « littérature » comme "langue hors pouvoir, dans la splendeur d'une révolution permanente du langage» (Barthes, 1978: 804); un poète que Michel Butor dévoile sous ce que le même auteur n'hésite pas à appeler un dogmatisme ${ }^{3}$, comme un contradicteur volontaire, comme celui qui a pleine conscience de ce qui ne peut passer dans l'objet écrit : ses silences ; un poète qui conçoit l'acte d'écrire (et je cite encore Butor) comme celui de « remettre aux autres de fermer eux-mêmes [sa] propre parole, et l'écriture n'[étant] qu'une proposition dont on ne connaît jamais la réponse » (Butor, $1974: 376$ ).

Un troisième sous-titre nous permettrait de le situer, en tant que lecteur de la ville, «entre acte d'écrire et pratique sociale »4, y assumant le rôle de «logothète » (Barthes, 1974a : 72), d'intellectuel - « être de langage »-, dans la civitas (au sens propre de « corps social de la cité »), les citoyens ; une sociabilité qui a aussi une dimension érotique pour Barthes, la ville étant le « lieu de rencontre avec l'autre ». Il emploie d'ailleurs dans cet article les expressions «érotique» et «socialité " «indifféremment» (Barthes, 1967 : 445), assumant dans la ville le rôle d'écrivain, l'écrivain étant le "sujet d'une pratique » (Barthes, 1978: 807) et "l'activité écrivante [s'insérant] parmi les autres activités sociales $»^{5}$ (Barthes, $\left.1974: 69\right)$ : l'écrivain étant celui qui prend parfois la liberté « de créer des néologismes $»^{6}$ (Barthes, 1974a : 73). Butor le considérera «le plus fécond forgeur de mots (...) l'un des plus grands de toute notre littérature » (Butor, 1974: 383), celui qui déplace le langage, "guetteur qui est à la croisée de tous les autres discours, en position triviale par rapport à la pureté des doctrines ${ }^{7}$. En position triviale comme Baudelaire, à ce carrefour de Paris où il perdra son auréole..., l'écrivain pour qui 'lire c'est écrire', pour qui « rendre la lisibilité au texte c'est écrire sur ce texte, ou bien le récrire, en faire parfois une lecture irrespectueuse, annonçant ainsi, peut-être, une posture postextualiste ${ }^{8}$ à son insu.

Un quatrième sous-titre nous permettrait de le situer en quête du référent qu'il a banni lui-même de la théorie dans les années 1960-1970², mais auquel la littérature ne saurait que très difficilement renoncer, sous peine de perdre sa consistance (sinon ses lecteurs), un sous-titre qui nous permettrait d'ouvrir la notion de logothète - qu'il ne conçoit pas comme « un écrivain qui invente des mots [mais comme] quelqu'un qui sait voir dans le monde, dans son monde (...) des éléments, des traits, des 'unités' (...) qu'il combine et agence d'une façon originale, comme s'il s'agissait d'une langue nouvelle dont il produirait le premier texte» (Barthes, 1974a: 73), allant finalement au-delà de ses perspectives strictement linguistiques, que les tendances du «retour au réel» contemporain de la théorie et de la littérature, nous invitent à nous interroger devant les nouveaux réalismes qui la caractérisent. La littérature peut-elle dire le monde ? Comment peut-elle le faire ? Ce sont des questionnements ouverts par le structuralisme, mais que la tendance du « retour au réel » contemporain, réactualise. Importants dans le cadre d'une réflexion sur la pensée de Barthes au sujet de la ville comme langue, comme espace sémiologique, le concept de "paysage de ville », ou celui de "paysage urbain » ont été travaillés, entre autres, par Françoise Chenet-Faugeras, pour qui « la ville et ses paysages constituent le vrai sujet du roman du vingtième siècle » (Chenet-Faugeras, $2007: 38)^{10}$, ou par Pascal Sanson ${ }^{11}$, qui considère ce dernier comme un «concept polysémique, aux 
dimensions plurielles, [qui] procède d'une redécouverte des dimensions signifiantes des espaces habités", des "multiples sémioses " qui jouent lors de l'appréhension d'un paysage (Sanson, 2007). Pour Michel Collot, se rapportant à la phénoménologie, et, en particulier à celle de Merleau-Ponty, qui lui permet de réunir «le sensible et l'intelligible » (Collot, $2011:$ 19), la collaboration entre littéraires, urbanistes, architectes, 'jardiniers' de la ville, les réunit autour d'une pensée-paysage qui inscrit la ville dans une logique de la géographie humaine, aux tonalités écologiques, mais aussi éthiques, qui créent de nouvelles urbanités. Le paysage est, pour Michel Collot «le produit de la rencontre entre le monde et un point de vue » (Collot, 2011:18): il devient par là un " enjeu stratégique »; pour Pascal Sanson, le concept de paysage urbain institue la ville comme un espace interdisciplinaire, l'inscrivant «dans une logique de la forme [pour] que la ville ait un sens pour ceux qui l'habitent et pour ceux qui la regardent » (Sanson, 2007 : 6). Voilà, me semble-t-il, ample matière à réflexion dans ce domaine, concevant des systèmes de signes que l'urbaniste se doit d'interpréter en vue de leur signification (et actualisant, pour employer l'expression d'Yves Citton (Citton : 2007), le souhait premier de Barthes dans l'article de référence, la possibilité d'une sémiologie urbaine.

6 Lire Barthes aujourd'hui nécessite qu'on soit attentif à cela à quoi a donné lieu la pensée barthésienne ; selon une posture réceptionnelle donc: par les effets, les prolongements, par la voix des héritiers, et en essayant de dépasser la canonisation dont l'oeuvre a fait l'objet (Compagnon, 1998: 12), de dépasser la seconde main dont il joue les offices dans tout article littéraire qui se doit. C'est aussi le lire dans ses textes originaux, en essayant de l'écouter dans le dialogue qu'il a pu entretenir avec lui-même, avec son œuvre et dans le dialogue que son œuvre a pu entretenir avec lui, et avec elle-même.

Or, lire Barthes en ce moment, c'est aussi situer notre lecture de Barthes à un moment où «un débat théorique est (...) en cours entre les tenants du retour au référent et les tenants de la littérarité » (Roelens, $2014: 59$ ).

La réflexion de Barthes s'inscrit en plein tournant linguistique, sa conférence datant de 1967, tournant marqué par les travaux de Saussure, en France, comme fondement de la théorie littéraire (Compagnon, 2005). Ses recherches s'insèrent à l'époque dans le cadre du structuralisme qu'il définit en ces termes, lors d'un entretien à la suite de la publication de Système de la Mode, la même année : «il s'agit d'un mouvement de pensée et d'analyse qui s'efforce de retrouver, par certaines méthodes extrêmement précises, la structure des objets sociaux, des images culturelles, ses stéréotypes, aussi bien dans les sociétés archaïques que dans nos sociétés modernes techniciennes » (Barthes, 1967a: 465) ${ }^{12}$.

9 Si les questions d'urbanisme ne semblent pas occuper une place considérable dans les articles de Barthes ${ }^{13}$, il faudra les situer dans le cadre de recherche décrit, et non comme des épisodes autonomes. Justement, sa réflexion sur le langage, sur les langages, qui l'a occupé toute sa vie durant, répond à cette quête de "méthodes extrêmement précises " que l'analyse structuraliste demandait et à laquelle la linguistique semblait être en mesure de fournir les instruments opératoires nécessaires. Pour sa part, et en toute cohérence dans ce cadre, il "s'est penché sur ces phénomènes de notre société qui sont (...) des ensembles d'objets utilitaires : les aliments, s'il s'agit de nous nourrir, les maisons, s'il s'agit de nous loger, les rues des villes, s'il s'agit de circuler, et la mode, s'il s'agit de nous vêtir » (Barthes, 1967a : 465). Barthes s'est ainsi particulièrement intéressé dans cet article aux langages qui déploient le tissu de la ville - qui devient un tissu textuel ${ }^{14}$ depuis ce « vêtement sans fin » qu'il aurait imaginé envelopper le corps féminin (Barthes, $1967: 170)^{15}$ - , à ces mots que Butor, à son tour, entend jaillir de partout, sous la forme 
orale ou écrite, qui justifient le titre de son article de 1982, "La ville comme texte ». L'« accumulation» de textes qui accompagnent Butor lors de son arrivée à « une ville étrangère ", en l'occurrence, la ville de Tokyo, fait se rassembler dans l'espace de son voyage les livres lus/écrits, en train d'être lus/écrits, ou qui le seront (Butor, 1982 : 33), le « texte manifeste » et le «texte profond, celui qui dort entre les feuilles d'un livre pas encore ouvert, aux souterrains des réserves (...)» (Butor, 1982: 36), ces textes, finalement, qui évacuent le référent et instituent un arrière-texte fécond (Gladieu et al., 2013). Celui-ci interpelle des connexions intertextuelles qui élargissent la conception de J. Kristeva de texte comme le résultat de la lecture d'autres textes, de la citation d'autres textes : « Tout texte se construit comme mosaïque de citations, tout texte est absorption et transformation d'un autre texte » (citée par Compagnon, 1998 : 117).

$\mathrm{Au}$ sémiologue de la mode s'offre un modèle de travail similaire à celui du sémiologue urbain : «Ce vêtement total, il faut l'organiser, c'est-à-dire, découper en lui des unités signifiantes, afin de pouvoir les comparer entre elles et reconstituer ainsi la signification générale de la mode. Ce vêtement sans fin a une double dimension: d'une part, il s'approfondit le long des différents systèmes qui composent son énoncé ; d'autre part, il s'étend, comme tout discours, le long de la chaîne des mots ; il est donc fait, ici, de blocs superposés (ce sont les systèmes ou codes), là de segments juxtaposés (ce sont les signifiants et les signifiés et leur union, c'est-à-dire, les signes) (Barthes, $1967: 170)$. De même que ce "vêtement sans fin " qui « se donne à travers un texte sans fin " comme le texte de la ville, de même conviendrait-il, pour Barthes, d'identifier les «unités signifiantes » de la ville, afin d'en esquisser leur « signification générale ». Effectivement, tel que l'a vu Butor, « ce n'est pas la mode elle-même qui l'intéresse, ce sont ses mots (...) Pour lui la mode est en quelque sorte toujours 'revêtue' d'un langage » (Butor, 1974 : 384), "le vêtement est un langage " (ibid., 385), de même que la ville est, pour Barthes, un langage, peut-être mieux, le carrefour de plusieurs langages: «celui qui voudrait esquisser une sémiotique de la cité devrait être à la fois sémiologue (spécialiste des signes), géographe, historien, urbaniste, architecte et probablement psychanalyste » (Barthes, 1967 : 439). "Spécialiste des signes », Barthes avoue ne l'être qu'«à peine »: «En fait, je ne suis rien de tout cela si ce n'est, et encore à peine, sémiologue » (ibid.). Sa vision de la ville sera superficielle, celle de la "surface », celle d'un " amateur », « au sens étymologique de ce mot: amateur de signes, celui qui aime les signes, amateur de villes, celui qui aime la ville» (ibid.); mais elle sera attentive à tous les signes dont se compose ce reflet du fond du texte/ville sur sa surface : les mots du livre/les signes de la ville ${ }^{16}$.

11 Entre 1966 et 1968, Barthes effectue plusieurs voyages au Japon ${ }^{17}$, notamment à Tokyo ; ces voyages, il a pleine conscience de les faire en tant que lecteur : « je suis là-bas lecteur, non visiteur ", fasciné par le langage de la ville (Barthes, 1970: 44). Comme le souligne Bertrand Westphal, Barthes inaugure avec L'Empire des signes, « un des grands chantiers de la théorie littéraire des années soixante-dix : la lecture de la ville » (Westphal, 2007 : 257).

Or Barthes écrit dans son article le manque d'attention, chez les urbanistes, aux espaces urbains comme étant des espaces de signification. Se rapportant aux villes de la Grèce ancienne, Roland Barthes précise qu'« à cette époque, on avait une conception de la ville exclusivement signifiante, car la conception utilitaire d'une distribution urbaine basée sur des fonctions et des emplois, qui prévaut incontestablement de nos jours, apparaîtra plus tardivement ». Il considère par la suite qu'« il est étrange que, parallèlement à ces conceptions fortement signifiantes de l'espace habité, les élaborations théoriques des 
urbanistes n'aient accordé jusqu'à présent (...) qu'une place réduite aux problèmes de signification » (Barthes, 1967 : 440). Cependant lui-même se limite, à cet égard, à signaler en note de bas de page à peine, la publication en 1965 de l'anthologie critique sur l'urbanisme contemporain, L'Urbanisme, utopies et réalités. Une anthologie, par Françoise Choay, architecte, théoricienne de l'urbanisme, de son histoire et de l'histoire du patrimoine ; publication très récente par rapport à sa conférence, mais d'une importance décisive dans ce domaine d'études.

Barthes aurait voulu contribuer à l'élaboration d'un modèle sémiotique pour penser l'urbanisme, en invitant les urbanistes à penser les significations de la ville. Les urbanistes, architectes, et autres professionnels devraient y répondre au tournant du xxI ${ }^{\mathrm{e}}$ siècle par une multifocalisation que la géocritique allait introduire comme socle de son analyse de l'espace, en littérature.

14 La sémiologie, conçue par Barthes comme méthode pour l'urbaniste, devrait effectuer le passage de la cité comme discours, au discours comme langage. Pour cela elle devrait effacer les traces de la métaphore » langage de la ville» (Barthes, 1967: 442) (utilisée aussi pour le « langage » des fleurs, du cinéma, etc.), elle devrait passer de la métaphore à l'analyse, "de la métaphore à la description de la signification », elle devrait effectuer le passage de l'analyse des microstructures de la ville à la description de ses macrostructures. Il lui faudrait, finalement, reconnaitre qu'il existe un « rythme » propre à la ville « du moment qu'elle est habitée par l'homme, et faite par lui », parallèlement au «rythme fondamental de la signification qui est l'opposition, l'alternance et la juxtaposition d'éléments marqués et d'éléments non marqués ». Cependant, la fin de cet article interroge, en quelque sorte, les certitudes de cette méthode.

Pour Barthes, « l'espace humain en général (et non seulement l'espace urbain) a toujours été signifiant» (Barthes, 1967: 439); des obstacles se dressent contre ce projet, que l'auteur identifie en termes de conflits, tout d'abord entre signification et réalité, celle de la "géographie objective, celle des cartes", dans le contexte de l'espace humain conçu comme espace géographique, pour conclure que « la signification est vécue en opposition complète aux données objectives» (Barthes, 1967 : 441). Dans Leçon, il reviendra sur la question de la représentation du réel par la littérature, qu'il désigne par sa «force de représentation » en soulignant l'« inadéquation fondamentale du langage et du réel », ce réel qui « n'est pas représentable - mais seulement démontrable », par les mots (Barthes, $1978: 806$ ).

16 S'intéressant à la notion de centre, qu'il envisage d'un point de vue historique à partir de la notion classique d'«Isonomie » (privilégier le centre), Barthes conclut sur l'existence d'un deuxième conflit, "entre la signification et la fonction »: la constatation par les urbanistes du "conflit entre le fonctionnalisme d'une partie de la cité, disons d'un quartier, et ce que j'appellerai son contenu sémantique (sa puissance sémantique) » (Barthes, 1967 : 441).

(fième conflit émerge, pour Barthes, entre «la signification et la raison (...) entre la signification et cette raison calculatrice qui voudrait que tous les éléments d'une cité soient uniformément récupérés par la planification » des urbanistes (Barthes, 1967 : 441).

Barthes fait appel aux facteurs de lisibilité de la ville, à partir d'une pratique de la ville, la ville étant habitée par l'homme tout autant que faite par lui.

L'élaboration d'une sémiologie urbaine consistera donc pour lui à « déchiffrer la ville » à partir d'« un rapport personnel », d'« une certaine ingénuité du lecteur » (Barthes, 1967 : 
446). La multiplication des lectures de la ville aboutira à ce que Barthes appelle «la langue de la ville ", et nous permettrait d'établir un parallèle éventuel avec ce qui allait devenir le projet d'une géopoétique de la ville: la quête de la langue d'une ville. Pour Michel Collot, ce néologisme, « inventé et revendiqué en France dans les années 1960 et 1970 par deux poètes » (Collot, $2014:$ 105), Michel Deguy et Kenneth White, « réunit deux ordres de réalités (...) : en traçant un trait d'union entre l'activité de l'esprit humain et l'espace, il remet en cause la distinction cartésienne entre la chose pensante et la chose étendue; et il enfreint les principes, structuralistes, de l'arbitraire du signe et de la clôture du texte, en supposant une certaine continuité entre l'expérience du monde et celle du langage » (ibid. : 105-106).

Bien que refusant la référentialité du langage, Barthes envisage à la même période la ville comme «structure mais qu'il ne faut jamais chercher/vouloir à remplir » (Barthes, 1967 : 446). La ville est donc pour lui un poème. Pas la ville classique organisée en conformité avec le concept grec d'«Isonomie», puisqu'il n'y a plus de centre aux villes modernes faites de décentrements; ceux-ci configurent la ville comme espace de l'imagination créatrice, espace qui se cherche une forme, qui change et demeurera toujours incomplet, au rythme de notre regard de flâneurs que nous sommes tous, tel que le reconnait Bertrand Westphal : "we only can catch a glimpse of reality, for a short while, modestly, as the flâneurs we all are, volens nolens, whether we like it or not » (Lévy \& Westphal, $2014: 188)$.

21 L'interrogation sur le référent est fondamentale en plein tournant linguistique, tournant textuel. Or Barthes nous invite à penser la ville comme une structure ouverte, dont les formes successives sont dictées par des facteurs extérieurs à la ville (ces référents que la sémiotique peircienne désignera par les representamen (cité par Roelens, 2014 : 58), par ces regards portés sur la ville par ses passants/lecteurs - écrivains. Barthes estime que la meilleure méthodologie pour connaître les villes c'est de les voir par les yeux des écrivains: «le plus important n'est pas tant de multiplier les enquêtes ou les études fonctionnelles de la ville que de multiplier les lectures de la ville, dont, malheureusement, jusqu'à présent, seuls les écrivains nous ont donné quelques exemples »; ces lectures permettraient de reconstituer une " langue », un « code » de la ville , tel qu'il le souhaitait Hugo comme l'un des premiers lecteurs de la ville. Ces regards deviendront à l'ère de la mondialisation et du tournant spatial les regards multiples, pluridisciplinaires ( multifocalisés) que la géocritique mobilisera dans son étude des espaces littéraires.

Les «structures" seraient-elles déjà, avec Barthes, sur le point de «descendre dans la rue »?

L'article de Barthes poursuit la recherche d'un modèle linguistique qui s'applique à d'autres disciplines, notamment les disciplines modernes sur la ville, comme l'urbanisme. Pour Barthes, sémiologue, il faut rester près du texte, considérer la ville comme texte; mais comme texte à lire: donc le besoin d'un lecteur, non pas celui dont Umberto Eco présentait une typologie dans Lector in fabula $(1979){ }^{18}$, ni celui que l'esthétique de la réception (que Hans-Robert Jauss développa en Allemagne dans ces mêmes années de 1960-1970) envisageait en termes sociologiques, mais d'un lecteur qui y lise les signes du modèle, qui y lise l'opacité du réel, l'effet de réel, l'illusion référentielle.

La langue émanant de cette analyse - la langue de la ville - était censée fournir un modèle valable pour le linguiste, pour le littéraire, pour l'urbaniste - unis dans un projet sémiologique commun, celui de rendre la ville lisible. 

au réel » qui justifie la popularité de perspectives interdisciplinaires qui font appel à la contribution d'autres savoirs. Ceux-ci redécouvrent l'intérêt de la littérature pour la connaissance des êtres humains, de leurs modes de vie, de leur vision du monde, ou bien pour la connaissance des habitats humains comme la géographie (on parle, dans cet encadrement, d'un tournant cartographique, d'un tournant spatial, de méthodes critiques géocentrées - comme la géocritique inaugurée par les travaux de Bertrand Westphal -, ou l'architecture et l'urbanisme, ou la philosophie (qui interroge des cartes incertaines (avec Alain Milon, 2011), l'écologie (avec Alain Suberchicot, 2012, ou Pierre Schoentjes, 2015 ${ }^{19}$ ), l'écologie qui ne saurait être isolée de soucis éthiques concernant le rapport des êtres humains au monde, leur responsabilité et la manière dont la littérature dit ce rapport.

ant les années 1980, «la réflexion sur la référence littéraire a été rouverte dans le cadre de la sémantique des mondes possibles ou fictionnels » (Compagnon, 1998: 142). Ce n'est plus que le monde fictionnel soit la copie du monde réel, mais que les mondes possibles « soient compatibles avec le monde réel » (ibid., 143). Et Compagnon insiste sur le besoin de dépasser « cette violente logique binaire, terroriste, manichéenne, si chère aux littéraires - fond ou forme, description ou narration, représentation ou signification " comblée par le couple mimétisme/antimimétisme, «alors que la littérature est le lieu même de l'entre-deux, du passe-muraille » (ibid. : 145).

Un autre sous-titre donne une pertinence à cette réflexion, «Barthes en espace urbain » : «lire Barthes après le retour du référent", en parallèle à sa croyance "peut-être avec quelque présomption, en la possibilité d'une sémiotique de la cité » (Barthes, 1967 : 439). La référence à l'espace urbain n'est peut-être chez lui qu'une provocation, étant donné sa référentialité immédiatement reconnaissable : un espace concret, la ville, et que Barthes rejette « toute hypothèse référentielle dans la relation de la littérature et du monde, ou même du langage et du monde » (Compagnon, 1998 : 115-116).

Pour Bertrand Westphal, « la quête de la lisibilité urbaine et spatiale n'est pas la marque d'une nouvelle forme d'exotisme paradoxale et postmoderne, vouée à la lecture de l'illisible» (Westphal, 2007: 257). La lisibilité de l'espace, inaugurée par Barthes, ne s'arrête pas aux discours que le livre tient sur la ville : «Ce n'est plus le livre et le discours par lui véhiculé qui viennent conforter l'examen de la ville perçue, mais au contraire la ville qui est coulée dans un discours qui tient lieu de matérialité, qui tient lieu, qui fait le lieu » (Westphal, $2007: 258$ ).

Actuellement, et il faudrait citer ici le livre d'Edward Soja, Thirdspace: Journeys to Los Angeles and Other Real-and-Imagined Places (1996), le rapport entre le texte et la ville, entre le texte-ville et le réel se conjuguent selon une poétique autre: «la déconstruction du concept traditionnel d'espace urbain, voire de lieu, entraîne une relation éminemment problématique entre ce qu'on qualifie de 'réel' et la discursivité. Elle consacre le statut de simulacre de certains espaces dans lesquels, comme a essayé de montrer Jean Baudrillard, le réel est supplanté par un hyperréel déréalisant»; la terminologie typologique des villes change, et Westphal en dresse la liste: «les Outer Cities, les Edge Cities, les Technopoles, les Technoburbs, les Silicon Landscapes, les Postsuburbia ou encore les Metroplex (...) autant de vocables alimentant une poétique postmoderne (post-humaine?) de l'urbanité des marges » (Westphal, 2007 : 259). Des espaces « anti-anthropomorphiques », comme ceux qui caractérisent les sociétés urbaines déshumanisées dans le récit Ano 1993, de Saramago, à la merci de la « singularité » technologique, de la robotisation, du clonage, peuplées par des êtres mi-humains, mi-mécaniques, soumis à des phénomènes d'auto-

Carnets, Deuxième série - 6 | 2016 
replication. Des espaces sans doute anticipés par le déphasage entre la carte et la géographie du souvenir dans le conte de Michel Tournier, La Fin de Robinson Crusoé, la carte et le territoire de Houellebecq, certainement par les conflits que Barthes décrivait comme des obstacles au travail du sémiologue, lecteur de la ville.

A l'époque où Barthes intervient sur ces questions, la critique met en cause toute une tradition de la «représentation» littéraire. Or l'auteur attire l'attention, dans "Sémiologie et urbanisme", sur la "prise de conscience croissante des fonctions des symboles dans l'espace urbain» (Barthes, 1967: 440). Il cite le conflit entre la " signification et la réalité ", c'est-à-dire, entre le langage et le réel, le conflit entre la "signification et la fonction" comme un des principaux problèmes des urbanistes contemporains (ibid., 441), et donne comme exemple la ville de Rome, marquée par le conflit entre « les nécessités fonctionnelles de la vie moderne et la charge sémantique qui lui est communiquée par son histoire "; le conflit "entre la signification et la raison » constitue un autre obstacle au travail de l'urbaniste, c'est-à-dire, entre la signification et la programmation des planificateurs de l'espace urbain, qui tendent à annuler les différences, à uniformiser. Barthes attire l'attention sur la nature inégale des villes : « une ville est un tissu formé non pas d'éléments égaux dont on peut inventorier les fonctions, mais d'éléments forts et d'éléments neutres, ou bien, comme disent les linguistes, d'éléments marqués et d'éléments non marqués (on sait que l'opposition entre le signe et l'absence de signe, entre le degré plein et le degré zéro, constitue un des grands processus de l'élaboration de la signification)»(Barthes, 1967 : 441).

Le rythme de la ville provient à son tour pour Barthes du «rythme fondamental de la signification qui est l'opposition, l'alternance et la juxtaposition d'éléments marqués et d'éléments non marqués », comme dans une phrase, comme dans la langue. Pour lui, « la cité est un discours, et ce discours est véritablement un langage : la ville parle à ses habitants, nous parlons notre ville, la ville où nous nous trouvons, simplement en l'habitant, en la parcourant, en la regardant " (Barthes, $1967: 441$ ). Barthes en espace urbain, c'est aussi l'homme des foules: c'est aussi celui qui jette son auréole, qui dérive, cet antimoderne qui est "sans cesse à la recherche d'une 'tierce forme' inclassable ", celui qui « réussit à imposer son ambiguïté, son 'atopie'» (Compagnon, 2005 :447). Celui qui cherche son thirdspace, sans doute, celui qui cherche sa place dans la ville, espace de toutes les expériences de la modernité et de la postmodernité, la ville où il part à la recherche de la lisibilité du poème, réduit à l'essentiel, le haiku, forme suprême d'exigence envers la langue, et la poésie, pour sauver le langage et le monde. Forme la plus belle d'engagement politique, d'intervention dans la polis - conçue comme civitas -, pour donner un sens à l'action humaine, dans ce qu'elle a d'essentiel, le langage, dans sa pureté, expurgé de l'allégorie (qu'il critiquait dans La Peste, de Camus). Barthes en espace urbain est aussi celui qui pressent, au long de ses dialogues avec Maurice Nadeau ou avec Claude Jannoud, déjà cités, que la littérature "va à sa perte », qu'il sait que le public est « fragile », et que « la culture de masse (...) n'est pas littéraire »; celui qui est nostalgique face à la disparition du "mythe de l'écrivain", des grands écrivains, des "grands mainteneurs de littérature», comme Valéry, Gide, Claudel, Malraux; celui qui fait référence aux choix passéistes du Prix Nobel ${ }^{20}$ (Barthes, 1974 : 64-72). Intéressante pour notre propos s'avère, la conscience de Roland Barthes sur le lien intrinsèque entre les formes littéraires et la vie des sociétés, qui changent et ce changement peut entraîner, du côté de la littérature, le changement des « conditions de production, de consommation et d'écriture ». Pour Barthes, "une société sans littérature est parfaitement concevable» 
(Barthes, 1974 : 72). Jules Verne l'avait prévu, dans son roman d'anticipation Paris au XXI $s_{i e ̀ c l e}{ }^{21}$, une ville où les bibliothèques littéraires auraient été remplacées par des bibliothèques scientifiques, techniques...

Pour Westphal, l'espace urbain devient « intertexture»: «Calvino fait Paris, Butor fait Tokyo qui fait Butor, Borges fait Buenos Aires, et ccetera, à l'infini » (Westphal, 2007 : 264). Face à la contemporanéité postmoderne, Westphal fait appel à une nouvelle dialectique entre l'espace et le réel. En effet, des «logiques communes", qui ne sont pourtant pas "identiques", mais "analogues ", régissent les récits contemporains et les «espaces humains » : le récit ne progresse plus linéairement; les espaces humains ne se définissent plus en termes d'opposition entre «centre» et "périphéries». Tous deux se complexifient et se diversifient. Baudrillard parlait de "simulacre », Steiner de la société du spectacle et de l'image pour dire le contemporain, Saramago construisait des clones, faisait référence aux formes artificielles de reproduction des humains. Et Westphal de conclure : «la déréalisation de l'espace entraîne sa fictionnalisation » (Westphal, 2007 : 264). A son tour, le récit offre de «nouvelles approches » du réalisme, bien éloignées du réalisme balzacien ou zolien: les nouveaux réalismes sont à saisir dans «la fictionnalisation généralisée [qui] introduit nécessairement la littérature et les autres arts mimétiques dans un ordre original, qui suppose une nouvelle approche du réalisme » ( ibid. : 264) : les frontières entre le réel et la fiction s'affaiblissent.

Comment caractériser ce nouveau réalisme? Pour Westphal, « le réalisme postmoderne est tout entier dans la représentation d'un monde qui se caractérise par une déterritorialisation accélérée, un monde éminemment 'transgressif'» (ibid. : 264). Une réalité de plus en plus proche de l'univers fictionnel. Peut-être pourrions-nous conclure notre lecture de l'article « Sémiologie et urbanisme » en considérant la possibilité, chez Barthes, d'une sémiologie fondée sur l'affectivité qui le lie aux espaces de la ville : Barthes aime la ville, il aime les signes. Marcel Roncayolo, spécialiste des études urbaines, rappelle l'étymologie du mot urbanité : pour lui « ce ne sont pas les choses qui sont dotées d'urbanité mais la conduite des hommes entre eux». Une étymologie dont le sens est attesté depuis le $\mathrm{xv}^{\mathrm{e}}$ siècle dans le Trésor de la Langue Française, où « le mot s'attache aux 'relations sociales entre habitants d'une ville'» (Sanson, 2007 : 25). Le rapport social de Barthes à la ville est de l'ordre de l'affectivité (il aime les villes), et de l'érotisme (comme rapport de social : la socialité). Urbanité va de pair avec « civilité, courtoisie, politesse » ( ibid. : 25). Le sens actuel d'urbanité, urbanisme - formes de la ville - est récent, tel que le souligne Roncayolo, mais il n'en exclut pas le sens classique.

Barthes en espace urbain c'est aussi celui qui, dans sa Leçon inaugurale au Collège de France (1977), dix ans après l'article "Sémiologie et urbanisme », avoue son désir d'écrire un roman, pour «dire ceux qu['il] aime » (Barthes, 1978: 835). Or il a accepté de parler d'urbanisme en sémiologue parce qu'il aime les signes, il aime la ville; alors que l'« instance [du roman] est la vérité des affects» (Barthes, 1978 : 835). On y lit la même posture qu'il avait adoptée pour dire la ville, en amateur; il adopte la même « méthode »: "Je me mets (...) dans la position de celui qui fait quelque chose » (ibid.) - l'urbaniste ne fait-il pas la ville ?; le sémiologue veut lui transmettre un modèle linguistique pour lire la ville, puis pour la faire - « et non plus de celui qui parle sur quelque chose : je n'étudie pas un produit, j'endosse une production; j'abolis le discours sur le discours; le monde ne vient plus à moi sous la forme d'un objet, mais sous celle d'une écriture » (ibid.). Il s'agit d'écrire la ville, la ville comme texte, ajoute-t-il - « c'est-à-dire (...) une pratique » (ibid.). 
Barthes se situe donc « entre acte d'écrire et pratique sociale »- « je passe à un autre type de savoir (celui de l'Amateur) et c'est en cela que je suis méthodique » (ibid.).

Barthes ne manque pas de nous interpeller à l'heure actuelle : il nous invite à dire la ville sous plusieurs angles d'approche, ce que j'ai essayé de montrer ici par un florilège de sous-titres. Il faudra le lire en sémioticien et en poète et en critique en quête du référent perdu. Qu'est-ce que le paysage urbain? Quel rapport entre l'architecte et l'urbaniste? Quelle pensée du paysage? Prendre en compte Barthes en espace urbain, c'est ouvrir d'autres perspectives sur la question de lecture de la ville, des villes, des villes d'aujourd'hui. Il y aurait, du reste, bien d'autres sous-titres à ajouter à ces propositions.

\section{BIBLIOGRAPHIE}

BARTHES, Roland (1964). «Réflexion sur le style de L'Etranger », in (1993) Roland Barthes, CEuvres complètes, (1942-1965), Paris : Seuil, t. 1.

BARTHES, Roland (1964). «Qu'est-ce que la critique ? ", in (1993) Roland Barthes, Euvres complètes, t. I (1942-1965). Paris : Seuil.

BARTHES, Roland (1967). « Sémiologie et urbanisme », in (1994) Roland Barthes, Euvres complètes, t. II (1966-1973). Paris : Seuil.

BARTHES, Roland (1967a). Système de la mode. France-Forum, 5 juin 1967, propos recueillis par Cécile Delanghe, in (1994) Roland Barthes, Euvres complètes t. 2 (1966-1973). Paris : Seuil.

BARTHES, Roland (1970). L'Empire des signes. Paris : Flammarion

BARTHES, Roland (1974). « Ou/Ou va la littérature? » Dialogue avec Maurice Nadeau, in (1995)

Roland Barthes, CEuvres Complètes (1974-1980), Paris : Seuil, T 3.

BARTHES, Roland (1974a.). « Roland Barthes contre les idées recues », Propos recueillis par Claude Jannoud, in (1995) Roland Barthes, CEuvres Complètes (1974-1980). Paris : Seuil, T. 3

BARTHES, Roland (1978). « Leçon », in (1995) Roland Barthes, EEuvres Complètes (1974-1980). Paris : Seuil, T. 3

BUTOR, Michel (1974). «La Fascinatrice », Repertoire IV. Paris : Les Éditions de Minuit.

BUTOR, Michel (1982). «La ville comme texte », Répertoire V. Paris : Les Éditions de Minuit.

CITTON, Yves (2007). Lire, interpréter, actualiser. Pourquoi les études littéraires. Paris : Éditions Amsterdam.

CHENET-FAUGERAS, Françoise (2007). « Du paysage urbain », in Pascale SANSON, Pascal (dir.). Le Paysage urbain : Représentations, Significations, Communication. Paris : L'Harmattan.

Collot, Michel (2011). La Pensée-paysage. Paris : Actes Sud/ENSP.

Collot, Michel (2014). Pour Une Géographie littéraire. Paris : Éditions Corti.

COMPAGNon, Antoine (1998). Le Démon de la théorie : Littérature et sens commun. Paris : Seuil.

COMPAGNON, Antoine (2005). Les Antimodernes : de Joseph de Maistre à Roland Barthes. Paris : Seuil. 
JOUVE, Vincent (1986). La Littérature selon Roland Barthes. Paris : Les Éditions de Minuit.

LÉvy Clément, WESTPHAL, Bertrand (éds.) (2014). La Géocritique : état des lieux / Geocriticism : a Survey . Limoges : PULIM.

MILON, Alain (2012). Cartes incertaines, Regard critique sur l'espace. Paris : Encre marine.

ROELENS, Nathalie (2014). « La géocritique mise au pas : voyageurs, flâneurs, piétons », in Levy, Clément, Westphal, Bertrand, Géocritique : état des lieux, Geocriticism : a Survey. Limoges : PULIM.

RONCAYOLO, Marcel (1990). La Ville et ses territoires. Paris : Gallimard, « Folio ».

SANSON, Pascal (dir.). (2007). Le Paysage urbain : Représentations, Significations, Communication. Paris : L'Harmattan.

schoentJes, Pierre (2015). Ce qui a lieu. Marseille : Wildproject. Editions, « Tête nue ».

SOJA, Edward (1996). Thirdspace: Journeys to Los Angeles and other real-and-imagined places. Oxford : Blackwell .

SUBERCHICOT, Alain (2012). Littérature et environnement. Pour une écocritique comparée. Paris :

Champion, « Unichamp-Essentiel ».

GLADIEU, Marie-Madeleine et al. (2013). L'Arrière-texte : pour repenser le littéraire. Bruxelles : P.I.E. Peter Lang.

WESTPHAL, Bertrand (2007). La Géocritique : réel, fiction espace. Paris : Les Éditions de Minuit.

\section{NOTES}

1. Conférence organisée par l'Institut français de l'Institut d'histoire et d'architecture de l'université de Naples (Barthes, 1967 : 446).

2. Antoine Compagnon, sur Barthes dans « son dernier cours au Collège de France de 1978 à 1980, La préparation du roman »: « Barthes s'y tourne de plus en plus vers la poésie comme seule chance de sauver la littérature dans un monde qui ne l'aime plus. Barthes antimoderne, c'est aussi un Barthes poète » (Compagnon, $2005:$ 404).

3. Pour Butor, Barthes a une "pensée fondamentalement ouverte», mais qui «s'exprime néanmoins sur un ton remarquablement dogmatique auquel elle doit certainement une part de son pouvoir de persuasion » (Butor, $1974: 375)$.

4. Expression empruntée au texte d'introduction aux propos recueillis par Claude Jannoud, dans son interview à Roland Barthes, « Roland Barthes contre les idées reçues », publiée dans Le Figaro, 27 juillet 1974 (Barthes, 1974a :70).

5. Tel que Barthes le soutient lors de son «dialogue avec Maurice Nadeau dans l'émission 'Dialogues', France-Culture, 13 mars $1974 »$ (Barthes, 1974 : 57-69).

6. Faisant référence à l'appellation de «logothète » que lui décerne Louis Calvet, dans l'article inséré dans le numéro 56 de la revue L'Arc qui lui est consacré en 1974 (un numéro réédité par ailleurs en 2007), Barthes soutient que «l'appréciation généreuse de Calvet se référait [...] à la liberté qu['il prend] parfois [...] de créer des néologismes » (Barthes, 1974 : 73).

7. Barthes précise l'étymologie latine de l'expression, la confluence de trois voies à l'origine : « trivialis, c'est l'attribut étymologique de la prostituée qui attend à l'intersection de trois voies » (Barthes, $1978: 807$ ).

8. A ce propos, cf. Schuerewegen, Franc, Introduction à la méthode postextuelle. L'exemple proustien, Paris : Classiques Garnier, "Théorie de la littérature », 2012. Et aussi, l'entretien avec Frank 
Wagner, publié le $1^{\text {er }}$ février 2013: http://www.vox-poetica.org/entretiens/ intSchuerewegen.html (consulté le 10 juin 2015).

9. Sous-titre possible : «à la recherche du référent égaré ». La question référentielle constitue l'une des préoccupations majeures de Barthes, cette question étant au centre de la réflexion de la théorie de la littérature française des années 1960-1970, qui incide surtout sur la problématique de la mimésis dans le roman réaliste français ; rappelons-nous ce recueil d'hommages qu'a été Littérature et réalité, publié en 1982 (Barthes étant décédé deux années auparavant), vrai florilège sur «l'effet de réel» (reprise de l'article de Barthes, 1968) «réaliste»; et sur l' «illusion référentielle» - , pour reprendre le titre de Michel Riffaterre (1978) qui y est republié, concernant la problématique plus vaste de la représentation du monde par la littérature (plus précisément par le poème, dans le cas de cet essai de Riffaterre) .

10. Architecte, actuellement professeur émérite à l'université de Tours. Maison des Sciences de l'Homme Val de Loire (MSH Vdl) ; Cités, Territoires, Environnement, Sociétés (Citeres).

11. Architecte, actuellement professeur émérite à l'université de Tours. Maison des Sciences de l'Homme Val de Loire (MSH VdL); Maison des Sciences de l'Homme Val de Loire (MSH Vdl); Cités, Territoires, Environnement, Sociétés (Citeres).

12. Le lien entre ce mouvement et la pensée de l'anthropologue Claude Lévi-Strauss est souligné par Barthes, entre autres textes, dans l'article «Qu'est-ce que la critique ?» donné au Times Literary Supplement en 1963, inséré plus tard dans Essais critiques: "on sait l'importance, on pourrait dire la vogue, de ce mouvement en France, depuis que Claude Lévi-Strauss lui a ouvert les sciences sociales et la réflexion philosophique » (Barthes, 1964 : 1357).

13. Nous citerions cependant, parmi ses essais, L'Empire des signes (1970), suscité par ses voyages au Japon, et Mythologies (1957).

14. La métaphore du texte comme tissu est récurrente dans l'œuvre de R. Barthes. Citons à propos ce passage de «Leçon »: «J'entends par littérature, non un corps ou une suite d'œuvres, ni même un secteur de commerce ou d'enseignement, mais le graphe complexe des traces d'une pratique : la pratique d'écrire. Je vise donc en elle, essentiellement, le texte, c'est-à-dire le tissu des signifiants qui constitue l'œuvre [...] Je puis donc dire indifféremment : littérature, écriture ou texte » (Barthes, $1978: 804)$.

15. Expression retirée du chap. 4 "Vêtement sans fin", de Système de la mode. Au sémiologue de la mode s'offre un modèle de travail similaire à celui du sémiologue urbain : « Ce vêtement total, il faut l'organiser, c'est-à-dire, découper en lui des unités signifiantes, afin de pouvoir les comparer entre elles et reconstituer ainsi la signification générale de la Mode »(Barthes, 1967 : 170).

16. Je renvoie à l'épigraphe placée en tête de cet article.

17. Cf. L'Empire des signes, Paris Flammarion, 1970.

18. L'Euvre ouverte datait déjà de 1962.

19. Nous tenons à remercier notre collègue Maria de Jesus Cabral pour nous avoir signalé cette publication récente.

20. Claude Simon se verrait accorder le Prix Nobel cinq années révolues sur la mort de Roland Barthes, en 1985.

21. Probablement écrit vers 1863, resté inédit jusqu'en 1994. 


\section{RÉSUMÉS}

Lire Barthes aujourd'hui c'est aussi écouter la voix des héritiers, et essayer de dépasser la canonisation dont l'œuvre a fait l'objet (Compagnon, 1998). C'est aussi lire Barthes dans le texte, en essayant de retrouver la profonde cohérence de sa pensée (Jouve, 1986). Nous nous proposons de réfléchir sur la possibilité que Barthes entrevoit d'une sémiologie urbaine, à partir du discours de plusieurs «spécialistes de signes". Cette approche devient autrement signifiante à un moment où un retour au réel intéresse la théorie littéraire contemporaine (Roelens, 2014).

Reading Barthes today implies a reader's response: looking for its "effects", his reception, hearing from him through those who have come after him, trying to go beyond his canonization (Compagnon, 1998). It also means to read his original texts looking for the coherence of his ideas (Jouve, 1986). In this article, we intend to reflect on the possibility of an urban semiology conjectured by Barthes, to which he invites the discourse of several "sign specialists". This approach is meaningful today when literary theory reconsiders the "referent" (Roelens, 2014).

\section{INDEX}

Keywords : Barthes (Roland), urban semiology, referent

Mots-clés : Barthes (Roland), sémiologie urbaine, retour au réel

\section{AUTEUR}

MARIA HERMÍNIA A. LAUREL

Universidade de Aveiro

hlaurel@ua.pt 